\title{
Weight Reduction of Tandem Compactor by Implementing Value Engineering Techniques
}

\author{
Jayachitra $\mathrm{R}^{1}$ \\ \{rjr.mech@psgtech.ac.in $\left.{ }^{1}\right\}$ \\ Department of Mechanical Engineering,PSG College of Technology, Coimbatore- 641004, \\ India.
}

\begin{abstract}
Tandem Compactor, a road construction machine is being developed at Larsen and Toubro Limited. The pilot machine of the tandem compactor is manufactured with the preliminary design. The total functional weight of the Tandem Compactor is $10.4 \mathrm{~T}$ which is above the target customer specification weight limit of $10 \mathrm{~T}$. This increase in weight adds up to the cost of the product. The increased cost of the product thereby increases the risk of losing the market share. This higher weight also deteriorates the machine fuel consumption and power utilization. Value Engineering technique aids in understanding the features and function of Tandem Compactor and to determine the functional weight of the existing system. Application of Value Engineering helps in achieving the Target Specification weight limit through design modification by finding creative concepts for achieving the desired function. The concepts are evaluated using several factors in order to develop a successful product and introduce it into the market. The modified system is verified and validated on the basis of functions it should perform, by comparing it with the existing system.
\end{abstract}

Keywords: Tandem Compactor; Value Engineering; Job Plan; Weight reduction.

\section{Introduction}

The market for road construction machinery in India is proliferating due to the increase in infrastructure development of the country. Vibratory Compactors are one among the road construction machinery which has huge market potential for 10T category but many local / global players could deliver product of the required specification with highly competitive price. The potential way to capture the market is by providing value to the customer with high level of product quality and lesser product cost. One of the ways of attaining this cost reduction is weight reduction.

The weight reduction in Tandem Compactor is achieved by a functional approach called Value Engineering (VE). VE does not reduce the cost of products by mere slashing of the price, but enhances their values through cost avoidance and improvement of functions. [1] VE is a customer oriented function-cost approach which focuses on customer demand.[2] VE can be applied during any stage of a project's design development cycle. However, the greatest are achieved early in the development and conceptual design stages. VE may be applied more than once during the life of the project.[3] VE uses several tools at various phases to obtain the desired results. Decision Matrix helps in judging ideas and alternatives [4]. Feature-Function Matrix, Function Cost worth Analysis helps in doing the functional analysis of the product [5]. 
Certain creative tools like TRIZ, Brain storming can be incorporated into VE process [6]. Interrogation can be done in order to speculate and evaluate [7]. VE helps in finding and removing the unnecessary cost in any project [8]. For optimizing a product or for evaluating its strength or performance software like ANSYS can be used [9].

Tandem Compactor is one of the machines used for making roads. The asphalt mix is transported using a truck and dumped into a paver. Paver then uniformly distributes the asphalt mix over a wide area; this laid asphalt mix is then compacted using Tandem Compactor [10]. The pilot machine of Tandem Compactor is made in L\&T- Product Development Centre. Testing is carried out in the pilot machine to validate the design criteria, performance and to ensure the operability of the machine as several assumptions are made during the initial design. Several tests are also carried out in order to identify the problems and overdesigned areas. The total functional weight of the Tandem Compactor is $10.4 \mathrm{~T}$ which is above the specification weight of $10 \mathrm{~T}$ which adds up to the cost. This increase in cost has the risk of losing the market share hence in order to reduce the cost of the machine the weight of the machine should be brought down. The other market gaining parameters are fuel consumption, compaction effectiveness, operator comfort and serviceability.

\section{Problem Statement}

The testing results of the pilot machine of Tandem Compactor revealed that the functional weight is $10.4 \mathrm{~T}$ which is above the target customer specification limit of $10 \mathrm{~T}$; this increase in weight raises the cost of the product. The increased cost of the product thereby increases the risk of losing the market share.

\section{Objective}

- To understand the feature and functions of Tandem Compactor and to determine the functional weight of the existing system.

- To achieve the target weight of $10 \mathrm{~T}$ from $10.4 \mathrm{~T}$ through design modification, by applying VE and to validate the modified system on the basis of its functions, by comparing it with the existing system before fiscal year- 2017.

\section{Methodology}

The methodology for Weight Reduction of Tandem Compactor by implementing VE Techniques is derived and the methodology flowchart is described in the Fig. 1. 


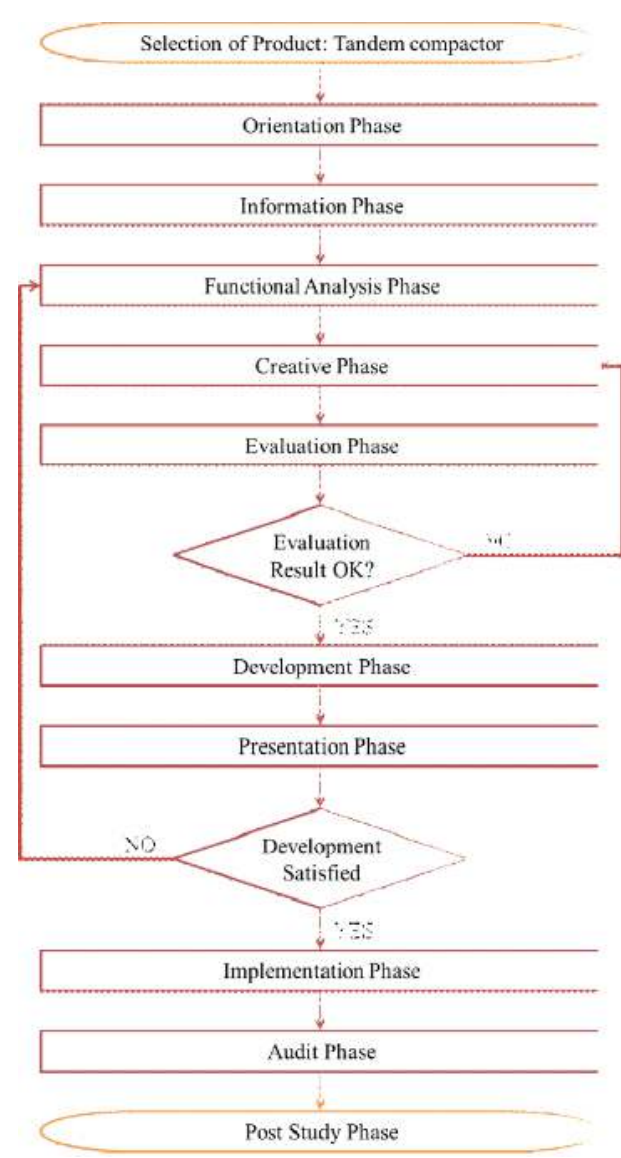

Fig. 1. Methodology Flowchart

\section{Selection Of Project}

Larsen and Toubro Limited, develops several machinery product one product amongst it is the Tandem Compactor. Pilot machine is made after the initial design. This machine has some issues which are to be solved before sending the product for serial production. Solving these issues is mandatory otherwise this product will not be commercialized. Hence this product is chosen for the project.

Tandem Compactor is in the design stage and its pilot machine is made to validate its performance against the specification. There are several parameters available to provide value to the customer such as increased fuel efficiency, increased power utilization, optimum compaction effectiveness, reduced cost etc. Weight reduction not only helps us to meet the specification but also helps us to attain some of the added values as mentioned above. But there are also some other negative impacts on weight reduction such as reduced robustness feel, reduced compaction effectiveness etc. 
In order to justify whether the weight reduction is appropriate or not, Force Field Analysis is carried out. Force Field Analysis analyzes all supporting and restraining forces, this helps in deciding whether to go about the project or not. The weightage for the supporting and restraining forces are given in the Table I. Higher the value higher is the impact.

\section{TABLE I. WEIGHTAGE FOR FORCE FIELD ANALYSIS}

\begin{tabular}{|l|l|}
\hline Description & Scale \\
\hline Very week & 1 \\
\hline Week & 2 \\
\hline Moderate & 3 \\
\hline High & 4 \\
\hline Very High & 5 \\
\hline
\end{tabular}

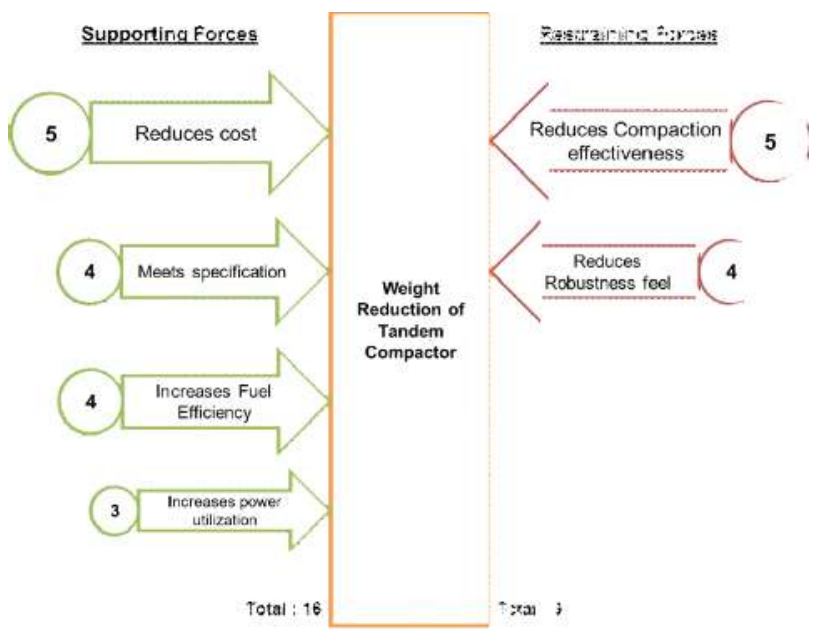

Fig. 2. Force Field Analysis

The total score for forces supporting The Weight Reduction of Tandem Compactor project is 16 , which is higher than the total score for forces restraining (9). Hence it is decided to carry out the project.

\section{Orientation Phase}

Orientation Phase is a very important phase in the VE study where the actual VE team was formed and the team is oriented. List of activities carried out in this phase are given below.

- Obtaining Senior Management Congruence and support

- Determining the Team Composition

- Orientation of team to Value Engineering

- Complete study on the Tandem Compactor- modules, Part list and Functions 
- Competitor Study

- Determining the Scope of the Project: Pareto Analysis A multi-disciplinary team is formulated, made cohesive and

they are introduced to the VE concepts and techniques.

Competitor study on the weight and cost details are made and the management decided to focuses on the $10 \mathrm{~T}$ category customer because of the high market potential.

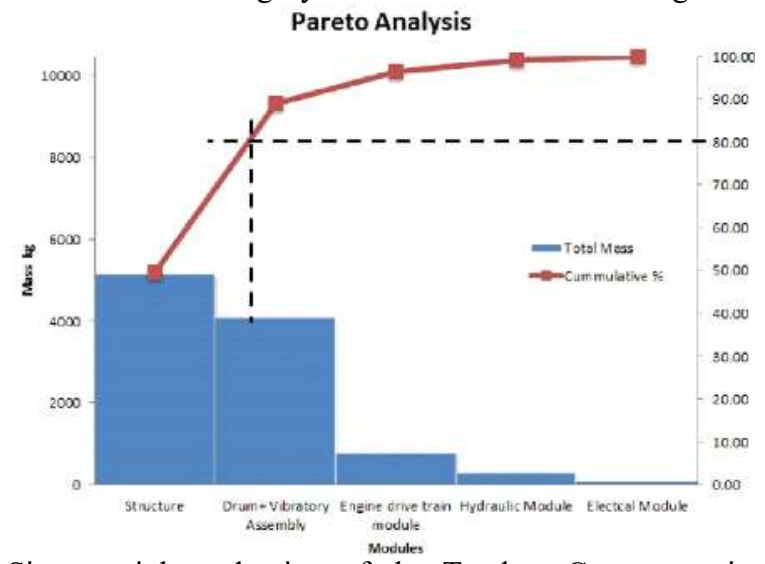

Since weight reduction of the Tandem Compactor is the objective, the mass of the individual assembly in every module is collected and a detailed analysis is carried out using Pareto chart to identify the scope of the project which is described in Fig. 3.

\section{Functional Analysis Phase}

Based on the inputs from the Information phase, Functional Analysis Phase is carried out separately for front chassis and rear chassis of chassis group. List of activities carried out in functional analysis phase are given below.

- Identification of Functions

- Classification of Functions

- Feature-Function Matrix

- Functional Analysis System Technique (FAST)

- Function-Cost-Worth Analysis (FCW analysis)

The functions of the front chassis and rear chassis as a whole are similar and it is described in Table II.

TABLE II. FUNCTION OF FRONT CHASSIS AND REAR CHASSIS

\begin{tabular}{|l|l|l|}
\hline Active Verb & $\begin{array}{l}\text { Measurable } \\
\text { Noun }\end{array}$ & Type \\
\hline Provide & Strength & Basic \\
\hline Accomodate & Structure & Necessary Secondary \\
\hline
\end{tabular}

Fig. 3. Pareto chart 


\section{Information Phase}

Information Phase is crux of the VE study where the actual data related to the product is collected and studied. Activities carried out in this phase are listed below.

- Collection of Module List

- Collection of Bill of Material

- Collection of Drawing

- Collection of 3D- CAD model

- Collection of Part details- Thickness, Material, Weight, etc.

- Compilation and study on Information

Based on the Pareto analysis the structure module of Tandem Compactor is initially taken for the VE Study. From the structures module the chassis group is initially selected for study. The details are described in the Fig. 4.

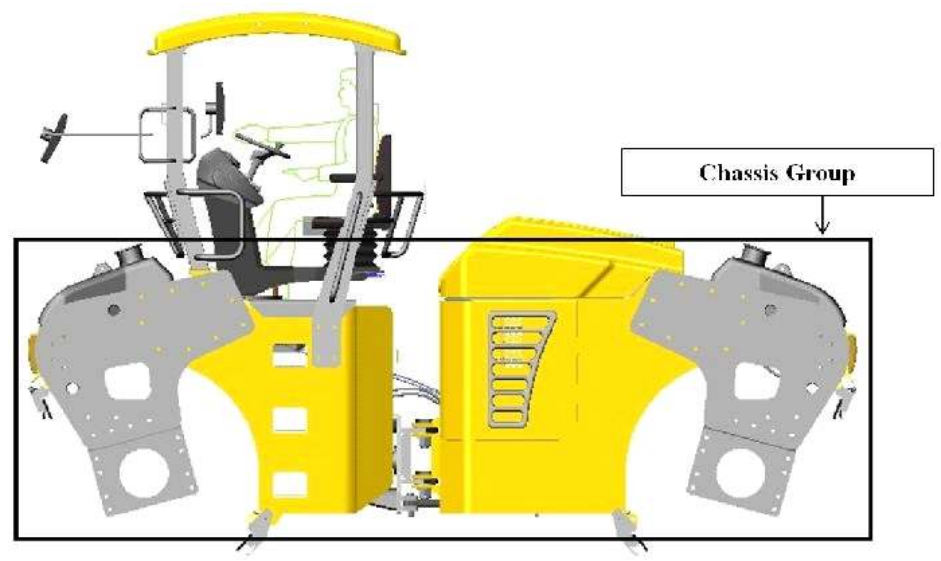

Fig. 4. Structures module of Tandem Compactor

The complete front chassis and rear chassis is segregated into assemblies; assemblies are segregated into sub-assemblies and sub-assemblies are segregated into parts. Then the features in the parts are identified and the function for the feature is identified and classified as basic and secondary functions. The Feature-Function Matrix is prepared for the same. The Feature Function Matrix for front chassis and rear chassis has 106 rows and 100 rows respectively due to the usage of several parts. From generated functions FAST diagram is drawn to pictorially analyze the functions. The FAST diagram of front chassis and rear chassis are shown in Fig. 5 and Fig. 6 respectively. 


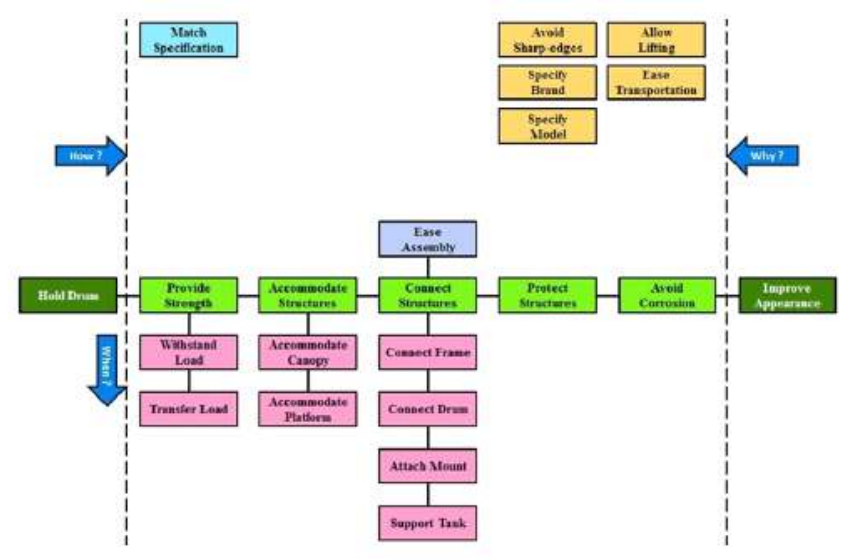

Fig. 5. FAST diagram of Front Chassis

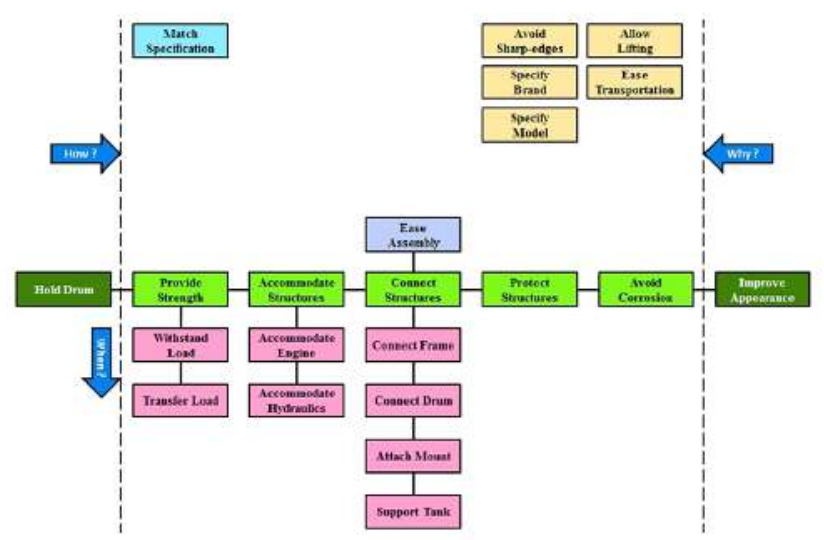

Fig. 6. FAST diagram of Rear Chassis

Cost per $\mathrm{kg}$ of material is Rs. 85 per $\mathrm{kg}$ which is inclusive of all costs. The weightages for cost allocation to function is done based on the features of the part. The weightages derived in congruence with the VE team and the cost is allocated to the function. There all several functions which are repeated for which the cost of the function is the sum of all the function cost. Function-Cost-Worth (FCW) analysis is carried out for both front chassis and rear chassis based on the cost allocated to it. The FCW analysis of the front chassis and rear chassis are described in the Table III and Table IV. 
TABLE III. FUNCTION-COST-WORTH ANALYSIS OF FRONT CHASSIS

\begin{tabular}{|c|c|c|c|c|c|c|c|}
\hline \multicolumn{2}{|c|}{ Eunowlinsate } & \multirow[b]{2}{*}{ 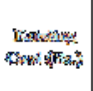 } & \multirow[b]{2}{*}{ Aisusuter } & \multirow[b]{2}{*}{ with } & \multirow[b]{2}{*}{ 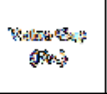 } & \multirow[b]{2}{*}{$\begin{array}{l}\text { Wels. } \\
\text { Therst }\end{array}$} & \multirow[b]{2}{*}{$x$} \\
\hline . & 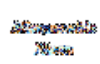 & & & & & & \\
\hline 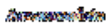 & 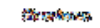 & Hand $20 x$ & 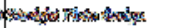 & 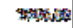 & 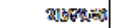 & Fos & 3 \\
\hline 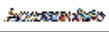 & sus & 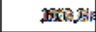 & 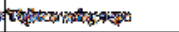 & litas & $\sin 2 x^{2}$ & 婹 & 4 \\
\hline 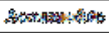 & 致; & $r+3$ & 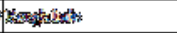 & 80 & 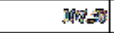 & 15.46 & 29 \\
\hline 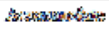 & 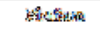 & $5 \times 9.50$ & 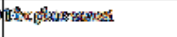 & sote & 새 & at: & ill. \\
\hline Mownededes: & $\csc x$ & shens & 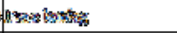 & Hisiss & 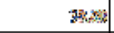 & es. & 346 \\
\hline Eváb & Monsa & 社部 & I I & 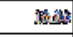 & 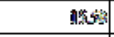 & 28 & 98 \\
\hline smonaxt & aturatres & 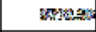 & 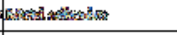 & 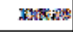 & MR: & $2 \pi$ & $x$ \\
\hline Wreanst & 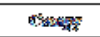 & 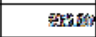 & 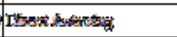 & 3 mans & 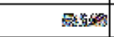 & 34 & 8 \\
\hline mandond & Preases: & 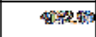 & 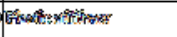 & $\operatorname{tin} x$ & 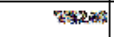 & 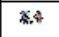 & 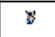 \\
\hline somost & $\sin x$ & nosectic & 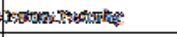 & 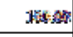 & 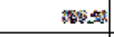 & 83 & 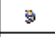 \\
\hline whorste & janto. & 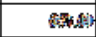 & 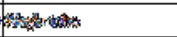 & sos & erk: & 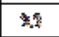 & is \\
\hline Hobirn & stusshars & somesise & theskententh & 158sto & weathos: & $\omega 3$ & 3 \\
\hline 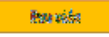 & 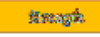 & $\sec x$ & 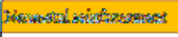 & 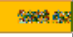 & Aheras & m: & 4 \\
\hline Hacerow: & $\operatorname{Tan}$ & Tasos & Thera dikwherst & жан & 清塔 & พิเ & $\mathrm{dY}$ \\
\hline Trensy & iati & 38: & 20: & 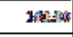 & 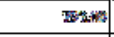 & *⿻ & 3 \\
\hline 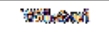 & Lentist & sant & 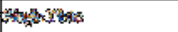 & most & $20 *$ & 24 & 309 \\
\hline
\end{tabular}

TABLE IV. FUNCTION-COST-WORTH ANALYSIS OF REAR CHASSIS

\begin{tabular}{|c|c|c|c|c|c|c|}
\hline \multicolumn{2}{|c|}{ Functions } & \multirow[b]{2}{*}{$\begin{array}{l}\text { Fxisting } \\
\text { Cost }(\mathbb{R} \text { s.) }\end{array}$} & \multirow[b]{2}{*}{$\begin{array}{l}\text { Worth } \\
\text { (Rs.) }\end{array}$} & \multirow[b]{2}{*}{$\begin{array}{c}\text { Vahuc Grap } \\
\text { (Rs.) }\end{array}$} & \multirow[b]{2}{*}{$\begin{array}{l}\text { Valer: } \\
\text { Index }\end{array}$} & \multirow[b]{2}{*}{ Rank } \\
\hline Activ Verth & $\begin{array}{c}\text { Measunable } \\
\text { Noun }\end{array}$ & & & & & \\
\hline Accormadars & Struta es & 20063.50 S Straigh- Plare design & 2000.00 & 180618.50 & 10.2 & 6 \\
\hline AccuLuxiali & How: & 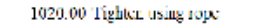 & 200.00 & $\$ 20.00$ & 10.2 & 5 \\
\hline Actom xiat: & Olizders & 306.00 Fis.l plats & 50.00 & 256.00 & 6.1 & 9 \\
\hline Connect & Structacs & 19295.001 meral arlicsive & 10000.00 & 1824.500 & 19.3 & 2 \\
\hline Coimed & Framies & A182.00 Pis lic stinteat: & 500.00 & $3+82.00$ & 8.1 & $\bar{z}$ \\
\hline t.annect & Motor & 'yul.s1) I3ortem liastening & 100.00 & etol..s & 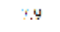 & $x$ \\
\hline Cormed & Druin & 663.00 Siggle tube & 200.00 & 463.00 & 3.3 & 11 \\
\hline Protesr & Structues & 1785.00 Prsatic Shoer & 150.00 & 1635.00 & 11.2 & 1 \\
\hline Proviti: & Sinzugilı & Y2367.00 Nurditil reinforcenucut & 2000.00 & 90367.00 & 46.2 & 1 \\
\hline Surpport & Tank & 1215.50 Face placeme it & 300.00 & 915.50 & 1.1 & 1.2 \\
\hline Thavin & 1..ranl & 174790 Sigl: link & $1000)$ & $164 ?: 01$ & 179 & i \\
\hline
\end{tabular}

From the FCW analysis it is inferred that the maximum value gap and value index are associated with the provide strength function. So this function should be concentrated for the design modification in Front Chassis and Rear Chassis.

\section{Creativity Phase}

From the Functional Analysis Phase it is understood that the major contributing function is 'Provide Strength'. Creative Phase aids to generate as many ideas as possible in order to provide creative solutions to provide strength. List of activities carried out in creativity phase is given below.

- Generating as many ideas as possible using Brainstorming Technique

- Selection of feasible ideas using Feasibility Ranking Technique

- Concept creation based on selected ideas

The ideas generated using the brainstorming techniques for the design modification of front chassis and rear chassis are shown in Table V. 


\begin{tabular}{|l|l|}
\hline S.No. & Ideas \\
\hline 1 & $\begin{array}{l}\text { Reduce chassis plate thickness } \\
\text { Change engine to motor }\end{array}$ \\
\hline 3 & Reduce Machine wheel base \\
\hline 4 & Reduce Chassis Width \\
\hline 5 & $\begin{array}{l}\text { Change suspended frame design by reducing } \\
\text { width } \\
\text { Reduce drum diameter }\end{array}$ \\
\hline 6 & Reduce Track Width \\
\hline 7 & Change to one drum \\
\hline 9 & Replace drum by flat press \\
\hline 10 & Track with compaction plate \\
\hline 11 & Integrated chassis \\
\hline 12 & $\begin{array}{l}\text { Reduce water tank volume } \\
\text { Reduce the no. of poles in canopy }\end{array}$ \\
\hline 13 & Reduce Hydraulic tank Volume \\
\hline 14 & Reduce Fuel Tank Volume \\
\hline 15 & Replace hose with tube \\
\hline 16 & $\begin{array}{l}\text { Replace 2 steering cylinder with 1 steering } \\
\text { cylinder }\end{array}$ \\
\hline 17 & Remove Lamp Beam \\
\hline 18 & Change material \\
\hline 19 & Change steering mechanism \\
\hline 20 & \\
\hline
\end{tabular}

TABLE V. IDEAS GENERATED USING BRAINSTORMING

\begin{tabular}{|c|c|c|c|c|c|c|}
\hline \multicolumn{2}{|c|}{ Fimerliuns } & \multirow[b]{2}{*}{$\begin{array}{l}\text { Existing } \\
\text { Cinst (Rs:-) }\end{array}$} & \multirow[b]{2}{*}{$\begin{array}{l}\text { Worth } \\
\text { (Rs.) }\end{array}$} & \multirow[b]{2}{*}{$\begin{array}{l}\text { Value Gap } \\
\text { (Rs.) }\end{array}$} & \multirow[b]{2}{*}{$\begin{array}{l}\text { Value } \\
\text { Tuder }\end{array}$} & \multirow[b]{2}{*}{ Kank } \\
\hline $\begin{array}{l}\text { Arfiver } \\
\text { Warb }\end{array}$ & $\begin{array}{l}\text { Howsurable } \\
\text { Namen }\end{array}$ & & & & & \\
\hline Accerracodiste & Sruetures & 23970.00 Seright Plate design & 2000.00 & 21970.00 & 12.0 & 2 \\
\hline Aromenatints & Those & $10>0$ no Tiplren usirg reppe & 10000 & 90000 & 102 & 1 \\
\hline Aresazevidale & $p_{\text {min }}$ & 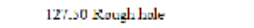 & 20.00 & $10 \% .20$ & 6.1 & 10 \\
\hline Accerrexjat: & Tlarforex. & 867.00 Flat plax mount & 200.00 & 667.00 & 4.3 & 11 \\
\hline Accorraxociate & Cancos: & 40.80 Tase buring & 10.00 & 30.80 & 4.1 & 12 \\
\hline Alt: h & Man-1 & 2,0 ineti huling & 1000 & 150 & 26 & 16 \\
\hline Coracest & Srustures & 9732.50 Mctal achesive & 1000.00 & 8732.50 & 9.7 & 5 \\
\hline Cornest & Cance: & 935.00 Direst fastering & 100.00 & 835.00 & 9.4 & 5 \\
\hline Cinnte: 1 & himus: & 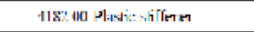 & צ110 on! & $382: 10$ & 81 & 8 \\
\hline Coracest & Motor & 790.30 3otcox r asteciurg & 200.00 & S95.30 & 9.9 & 9 \\
\hline Cornest & D.um & 665.00 Single tube & 200.00 & 463.00 & 3.3 & 15 \\
\hline Prote-t & Stmothuren & 1785 no Phatri- Sheet & 15000 & 153500 & 119 & 3 \\
\hline I'rovide & Straxist & 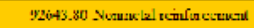 & 2000.00 & $905+3.80$ & 46.3 & 1 \\
\hline Sчррет & Tar: & 1215.50 Fase piacracat & 300.00 & 215.50 & 4.1 & 13 \\
\hline Trankfer & Thast & s9: no Siryte lirk & 1 ino on & $79 \div$ 保 & 89 & 7 \\
\hline Wiltivind & larad & 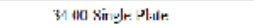 & 1000 & 2110 & 31 & 14 \\
\hline
\end{tabular}

The generated ideas are ranked using the feasibility ranking matrix; the factors used for ranking are shown in Table VI. 
TABLE VI. IDEAS GENERATED USING BRAINSTORMING

\begin{tabular}{|l|l|}
\hline Factor's Name & Factor \\
\hline F1 & $\begin{array}{l}\text { Congruence with specification } \\
\text { State of the art }\end{array}$ \\
\hline F2 & Probability of implementation \\
\hline F3 & Time required for implementation \\
\hline F4 & Cost of development \\
\hline F5 & Potential cost saving \\
\hline F6 & \\
\hline
\end{tabular}

The feasibility ranking matrix is shown in Table VII.

TABLE VII. FEASIBILITY RANKING MATRIX

\begin{tabular}{|l|l|l|l|l|l|l|l|l|}
$\begin{array}{l}\text { Idea } \\
\text { no. }\end{array}$ & F1 & F2 & F3 & F4 & F5 & F6 & $\begin{array}{l}\text { Total } \\
\text { Points }\end{array}$ & Rank \\
\hline 1 & 10 & 10 & 10 & 10 & 10 & 10 & 60 & 1 \\
\hline 2 & 1 & 1 & 1 & 1 & 1 & 4 & 9 & 17 \\
\hline 3 & 1 & 10 & 4 & 4 & 4 & 4 & 27 & 11 \\
\hline 4 & 10 & 10 & 10 & 10 & 10 & 7 & 57 & 2 \\
\hline 5 & 10 & 10 & 10 & 10 & 7 & 7 & 54 & 3 \\
\hline 6 & 1 & 4 & 4 & 4 & 4 & 4 & 21 & 13 \\
\hline 7 & 1 & 10 & 4 & 4 & 7 & 4 & 30 & 10 \\
\hline 8 & 1 & 1 & 1 & 1 & 1 & 1 & 6 & 18 \\
\hline 9 & 1 & 1 & 1 & 1 & 1 & 1 & 6 & 18 \\
\hline 10 & 1 & 1 & 1 & 1 & 1 & 1 & 6 & 18 \\
\hline 11 & 4 & 4 & 4 & 1 & 1 & 1 & 15 & 15 \\
\hline 12 & 10 & 10 & 10 & 10 & 4 & 4 & 48 & 5 \\
\hline 13 & 10 & 10 & 10 & 10 & 7 & 4 & 51 & 4 \\
\hline 14 & 4 & 10 & 7 & 7 & 7 & 4 & 39 & 7 \\
\hline 15 & 4 & 10 & 7 & 7 & 10 & 4 & 42 & 6 \\
\hline 16 & 7 & 4 & 4 & 4 & 4 & 1 & 24 & 12 \\
\hline 17 & 10 & 7 & 4 & 4 & 4 & 4 & 33 & 9 \\
\hline 18 & 10 & 10 & 4 & 4 & 4 & 4 & 36 & 8 \\
\hline 19 & 4 & 4 & 4 & 1 & 1 & 4 & 18 & 14 \\
\hline 20 & 7 & 1 & 1 & 1 & 1 & 1 & 12 & 16 \\
\hline
\end{tabular}

The highlighted ideas are selected for the concept generation and two concepts are created. The concepts are shown in Fig. 7.

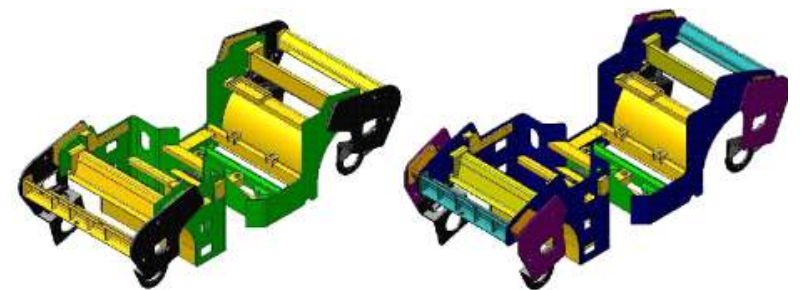

Fig. 7. Concept 1 and Concept 2 


\section{Evaluation Phase}

The concepts generated in the creativity phase are evaluated and suitable concept is chosen for development. List of activities carried out in Evaluation Phase are given below.

- Determining the Evaluation Factor

- Allocation of the weightage to the Evaluation Factor using Predetermined Minimum Method

- Evaluating idea using Evaluation Matrix

The factors upon which the concepts are to be evaluated are found by brainstorming with the VE team. The identified factors are listed in Table VIII.

\section{TABLE VIII. $\quad$ EVALUTION FACTORS}

\begin{tabular}{|l|l|l|}
\hline S.No. & Factors & Code \\
\hline 1 & Strength & A \\
\hline 2 & Material cost & B \\
\hline 3 & Manufacturability & C \\
\hline 4 & Manufacturing cost & D \\
\hline 5 & Ease of Assembly & E \\
\hline 6 & $\begin{array}{l}\text { Marketing team } \\
\text { response }\end{array}$ & F \\
\hline 7 & Aesthetics & G \\
\hline
\end{tabular}

Predetermined Minimum Method is used to assign weightage to the factors since it has the advantage of not ignoring any factors. After determining the point sharing based on weightage, minimum point allocation to factor and points per comparison, each factor is compared against all other factors; the complete paired comparison is shown in Fig. 8.

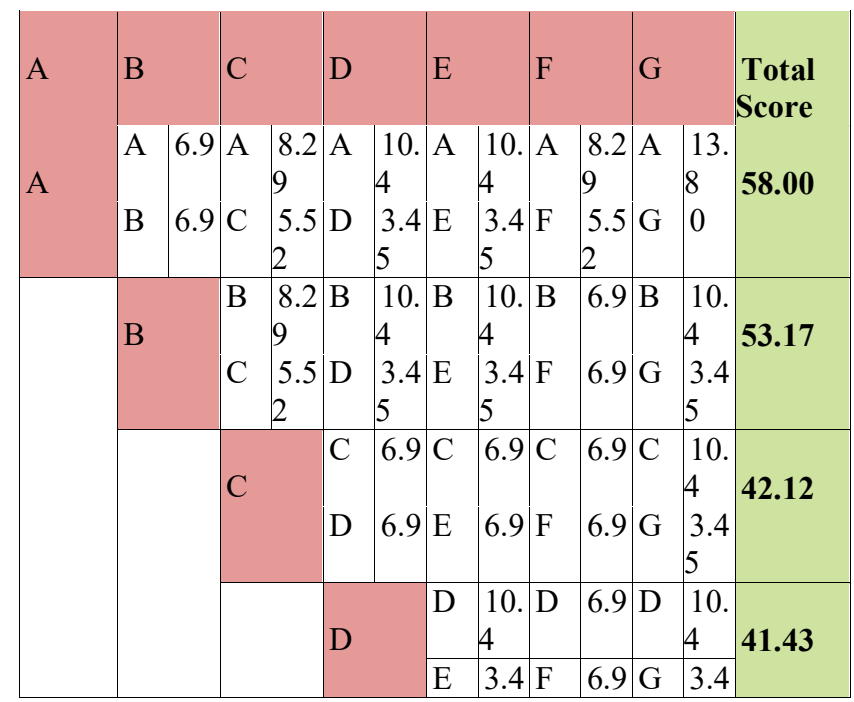




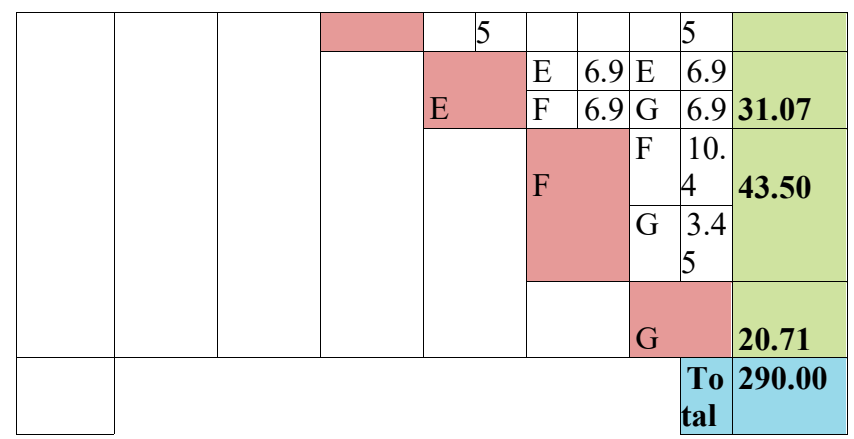

Fig. 8. Paired comparison

With the total score obtained from the paired comparisons the final weightage table is generated with the predetermined minimum points the description in shown in Table IX.

TABLE IX. FACTORS WEIGHTAGE

\begin{tabular}{|c|c|c|c|c|c|c|}
\hline t.s. & Mhotes: & Wedy & 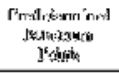 & 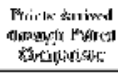 & 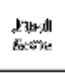 & 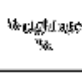 \\
\hline$T$ & Syanty & \$ & 3 & Fon & 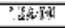 & $15 \$ 2 \%$ \\
\hline ז & 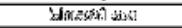 & 5 & Sas & $5 \times 5$ & 2.6 .211 & W:ils? \\
\hline$\%$ & ishaso hesoostants & 8 & सरा & $2, r \frac{1}{2}$ & 62.13 & 1425 \\
\hline$*$ & 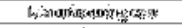 & r: & 20 & 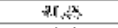 & $91, x$ & 19:3it \\
\hline$i$ & 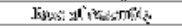 & $\mathrm{R}$ & 283 & $\$ \omega$ & $50 x_{2}$ & 19:pil \\
\hline is & 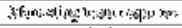 & $\mathrm{F}$ & $\pi i$ & 230 & ' 18.8 & $1 / 2,1$ \\
\hline it & 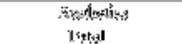 & $\mathrm{ir}$ & 年, & $x, 0$ & 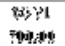 & 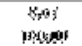 \\
\hline
\end{tabular}

From the factor weightage table it is inferred that the maximum weightage goes to the 'Strength' factor. The tool used to evaluate 'Strength' of the concepts is Finite Element Analysis (FEA). FEA is carried out for existing, concept 1 and concept 2 model for 4 load cases. Some result plots are described in Fig. 9, Fig. 10 and Fig. 11.

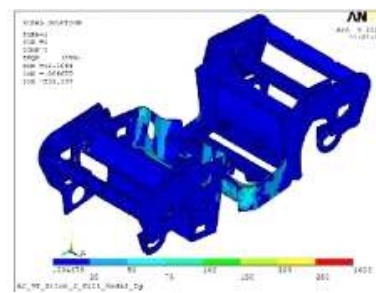

Fig. 9. FEA results for Existing machine

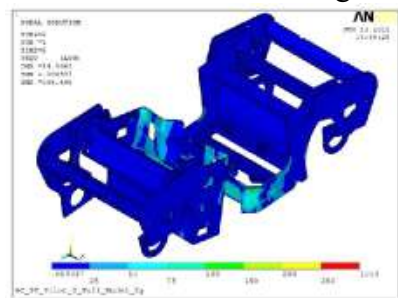

Fig. 10. FEA results for Concept 1 machine 


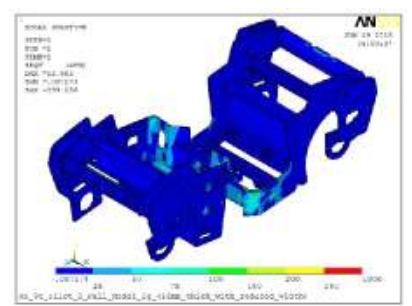

Fig. 11. FEA results for Concept 2 machine

'Five point' scale is used for the Evaluation. Other factors apart from FEA are evaluated with the congruence with the VE team and the persons from the areas of expertise. The Evaluation Matrix is shown in Table X.

\section{TABLE X. EVALUATION MATRIX}

\section{- DEVELOPMENT PHASE}

The Concept is finalized and the detailed design is prepared. The following activities are completed in the development phase and the final proposal is created.

- Design for Manufacturing and Assembly (DFMA)

- Detailed Drawings

- Engineering Change Notice (ECN)

- Estimate cost/ weight reduction

- Value improvement study

The estimated weight/ cost saving for a single Tandem Compactor is shown in Table XI. 
TABLE XI. COST / WEIGHT REDUCTION

\begin{tabular}{|l|l|l|l|l|}
\hline \multirow{2}{*}{ Assembly } & \multicolumn{2}{|l|}{ Strategy } & $\begin{array}{l}\text { Weight } \\
\text { Reduction } \\
\text { (kg) }\end{array}$ & $\begin{array}{l}\text { Cost } \\
\text { Reduction } \\
\text { (Rs.) }\end{array}$ \\
\cline { 2 - 5 } & Function & Cost & 17,862 \\
\hline Front Chassis & Maintained & Reduced & 210 & 18,008 \\
\hline Rear Chassis & Maintained & Reduced & 212 & $\mathbf{3 5 , 8 7 0}$ \\
\hline Total & & $\mathbf{4 2 2}$ & $\mathbf{2}$ \\
\hline
\end{tabular}

\section{Presentation Phase}

A detailed report is prepared including all the details about the concepts and the engineering analysis completed by the team during the VE study. The report is presented to the Management and stakeholders for review and approval. The Management and the stakeholders came to a common consensus that further improvements are required in order to further reduce the cost. This further cost reduction is necessary to capture high market share after product induction.

\section{Further Improvements}

Since further improvements are suggested the complete process from functional analysis phase to presentation phase is carried out for the canopy which is shown in the Fig. 12.

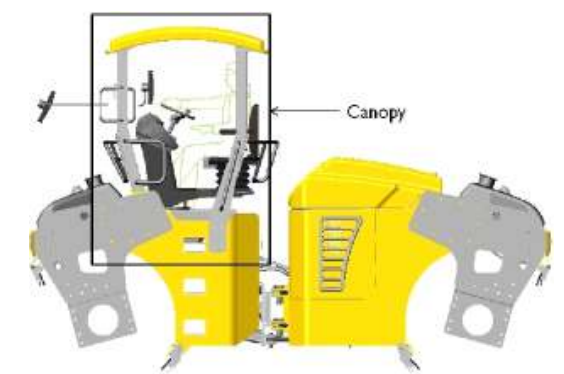

Fig. 12. Existing Canopy in the Structure module of Tandem Compactor

The new canopy thus developed which has only two poles with reinforcement in the top. The complete process is repeated as per the methodology and evaluation carried for the same factor. The final concept is approved and ready for implementation.

\section{- FINAL DEVELOPMENT PHASE}

The complete model is developed and presented to the management and proceed for implementation. The final machine is shown in the Fig. 13. 


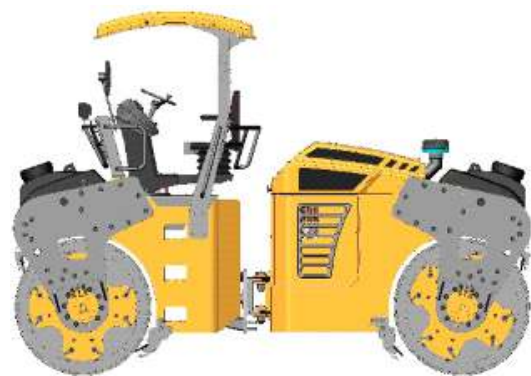

Fig. 13. New Tandem Compactor after Value Engineering

The cost and the weight reduction achieved after implementing VE is shown in Table XII.

TABLE XII. COST/ WEIGHT REDUCTION AFTER IMPLEMENTING VE

\begin{tabular}{|l|l|l|l|l|}
\hline \multirow{2}{*}{ Assembly } & \multicolumn{2}{|l|}{ Strategy } & $\begin{array}{l}\text { Weight } \\
\text { Reduction } \\
\text { (kg) }\end{array}$ & $\begin{array}{l}\text { Cost } \\
\text { Reduction } \\
\text { Rs.) }\end{array}$ \\
\cline { 2 - 5 } & Function & Cost & 210 & 17,862 \\
\hline Front Chassis & Maintained & Reduced & 18,008 \\
\hline Rear Chassis & Maintained & Reduced & 212 & 3,400 \\
\hline Canopy & Improved & Reduced & 40 & $\mathbf{3 9 , 2 7 0}$ \\
\hline Total & & $\mathbf{4 6 2}$ & \\
\hline
\end{tabular}

The final model thus developed not only has the benefit of reduced weight but also has improved visibility due to the removal of two poles in the operator cabin. This is a market gaining parameter and thus the value of the product is improved by this improvement.

\section{Implementation Phase}

After completing all the development activities the following activities are carried out.

- Final developed drawings are reviewed

- Final Drawings after review is approved and released

- The complete machine modification should be implemented

- The procurement of materials

- Creation of implementation plan

- Manufacturing and assembling

- Testing activities are to be carried out.

Machine modification implementation is approved and scheduled for the financial year of 2017. 


\section{Conclusion}

The Value Engineering principles and techniques are applied to the Tandem Compactor, a new product being successfully developed in Larsen and Toubro Limited. The features and functions of Tandem Compactor are completely studied and the functional weight of the existing machine is identified. Going through various phases of Value Engineering job plan a weight reduction of $462 \mathrm{~kg}$ per machine is achieved and the weight reduction achieved is validated using the CAD model. The functions of the modified objects are validated using the FEA in the evaluation phase, so the weight reduction is achieved without compromising the function. After manufacturing the new machine, both the functional and financial audits are to be carried out. There is still scope of further improvements in the field of operator comfort, serviceability, etc., which are to be identified and the next cycle for improvement should be initiated; since continuous improvement is not only the key to sustain in the market but also a vital element to grab a large market share.

\section{References}

[1] Anil Kumar Mukhopadhaya, "Value Engineering- Concepts, Techniques and Applications", Response Books, A Division of Sage Publications.

[2] IS 11810: 2003, Indian Standard, "Guidelines to Establish a Value Engineering Activity", Bureau of Indian Standards, December 2003.

[3] Amruta Chougule, A.K. Gupta and Sneha Patil, "Application of Value Engineering Technique to A Residential Building- Case Study", International Journal of Innovative Research in Advanced Engineering (IJIRAE), Volume 1, Issue 12, December 2014, ISSN: 2349-2163.

[4] Amit Sharma and Dr. R.M. Belokar, "Implementation of Value Engineering- A Case Study", International Journal of Marketing, Financial Servises \& Management Research, Vol.1 No.3, March 2012, ISSN 2277-3622.

[5] Chougule Mahadeo Annappa and Dr. Kallurkar Shrikant Panditrao, "Application of Value Engineering for Cost Reduction of Household Furniture Product- A Case Study", International Journal of Innovative Research in Science, Engineering and Technology, Volume 3, Issue 10, October 2014.

[6] Nitin B. Chaphalkar and Nilesh Agarchand Patil, "Study of Value Engineering Creative Phase for Highway Project by Using TRIZ”, International Journal on Emerging Technologies, 2011.

[7] Chougule Mahadeo Annappa and Kallurkar Shrikant Panditrao, "Application of Value Engineering for Cost Reduction- A Case Study of Universal Testing Machine", International Journal of Advances in Engineering \& Technology, July 2012, ISSN: 2231-1963.

[8] Seyed Ali Mousavi Dehmourdi, Professor. Ravande Kishore and Amin Alavi, "Value Engineering and Reason of Unnecessary Cost in Construction Industry", Australian Journal of Basic and Applied Sciences, 8(13) August 2014, Pages: 583-588, ISSN:1991-8178.

[9] Mandar Joshi and K A Rade, "Optimization of Weight of Cable Duct", International Journal of Engineering Research and General Science Volume 3, Issue 1, January-February, 2015, ISSN 2091-2730.

[10] Dipl. Ing. H.-J. Kloubert, BOMAG GmbH, "Basic Principles of Asphalt Compaction- Compaction methods Compaction equipment Rolling technique", BOMAG GmbH, Hellerwald, D-56154 Boppard, Edition 02/2009.

[11] S. Suhas, M.J. Silvister Raju, D.S. Vijayan, "Natural fibre reinforcement experimental study in polymer composite", Materials Today: Proceedings, , https://doi.org/10.1016/j.matpr.2020.12.051

[12] Lavanya Prabha, S., J. K. Dattatreya, and M. Neelamegam. "Investigation of bolted RPC plate under direct tension." Journal of Structural Engineering (Madras) 36.5 (2009): 333-341. 